\title{
EL MÉTODO DE COSTEO BASADO EN ACTIVIDADES Y SU INCIDENCIA EN LA FIJACIÓN DEL PRECIO EN LAS EMPRESAS AGROINDUSTRIALES VITIVINÍCOLAS
}

\section{THE ACTIVITY-BASED COSTING METHOD AND ITS IMPACT ON PRICE SETTING IN AGRO-INDUSTRIAL WINE COMPANIES}

\author{
Leticia Ruth Guillermo Vega ${ }^{1}$ \\ Evelyn Trinidad Montero Zúñiga ${ }^{2}$
}

Aceptado: 09/05/2020

Publicado online: $14 / 12 / 2020$

\begin{abstract}
RESUMEN
El presente estudio tuvo como objetivo determinar si la implementación de un método de costeo basado en actividades incide en la fijación del precio en las empresas agroindustriales vitivinícolas de la provincia de Tacna. Para implementar este método, se desglosó el proceso de elaboración del vino en actividades, se determinó los inductores de costo, que fueron asignados en función del nivel de uso de recursos por actividad. La implementación de este método demostró mayor exactitud y eficiencia en la asignación de costos de producción, al identificar el tipo de vino más costoso y el más económico, favoreció la reducción de costos, fue efectivo y personalizado para determinar el margen de ganancia por tipo de vino, en consecuencia, incidió en la fijación del precio por resultar más competitivo, real y diferenciado por producto. En contraste con el sistema tradicional, que proporcionó resultados distorsionados, al efectuar una distribución equivocada del costo en base al volumen de producción, fue inexacto en procesos con diferentes tipos de vinos, lo que generó diferencias considerables en los costos.
\end{abstract}

Palabras clave: $A B C$, costo, actividad, vino.

\begin{abstract}
The objective of this study was to determine whether the implementation of an activitybased costing method has an impact on price setting in agro-industrial wine companies in Tacna province. In order to implement this method, the winemaking process was broken down into activities, and cost drivers were determined, which were assigned according to the level of resource use per activity. The implementation of this method showed greater accuracy and efficiency in the allocation of production costs, by identifying the most expensive and the most economical by type of wine, favoring the reduction of costs, was effective and personalized to determine the profit margin by type of wine, consequently, influenced the setting of the price as it was more competitive, real and differentiated by product. In contrast to the traditional system, which provided distorted results, by making an erroneous distribution of the cost based on the volume of production, it was inaccurate in processes with different type of wines, which generated considerable differences in costs.

Keywords: $A B C$, cost, activity, wine.
\end{abstract}

\footnotetext{
${ }^{1}$ Maestro en Contabilidad Tributación y Auditoría. lerguillermo@upt.pe (ㄷ) 0000-0002-6931-2753

${ }^{2}$ Doctora en Ciencias contables y financieras. Magíster en Contabilidad - Auditoría. Contador público colegiado. emonteroz@unjbg.edu.pe @0000-0003-0761-1763
} 


\section{INTRODUCCIÓN}

Existen pocos antecedentes relacionados con la implementación de un método de costeo basado en actividades, como herramienta de gestión de costos aplicado a empresas manufactureras en elaboración de vinos. La problemática que afecta a estas empresas agroindustriales, reside en la determinación deficiente y arbitraria de los costos de producción, que en consecuencia conlleva a un bajo nivel de competitividad, las empresas pierden poder de negociación. Costos distorsionados generan precios equivocados; los costos tienen importancia estratégica, porque determinan precios y rentabilidades; el método de costeo tradicional ya no se adapta a las necesidades actuales, es limitado y presenta muchas deficiencias. El análisis de este problema, conduce a desarrollar una alternativa viable que responda en cierta medida a las necesidades actuales, a través del incremento de la productividad y competitividad, que impulse la producción de las empresas agroindustriales vitivinícolas de la provincia de Tacna; con relación a esto y desde un enfoque de la contabilidad de gestión; se considera necesario desarrollar un modelo o herramienta de gestión rigurosa y eficiente para determinar los costos, y tomar decisiones estratégicas en la fijación de precios, más aun cuando las empresas de este sector producen diferentes productos. De acuerdo con la Dirección Regional de Agricultura Tacna, a nivel de provincia, referente al cultivo de vid; registró una producción con tendencia de incremento de 5,172 toneladas de vid en el 2008, y de 6,408 toneladas de vid en el 2017. (Dirección Regional de Agricultura Tacna, 2017). Si bien la serie histórica de cultivos, refleja crecimiento en la producción de vid, el cultivo de uva para elaboración de vino aún es insuficiente.

Es fundamental entender la composición de los costos totales y unitarios, relacionados con el tipo de proceso productivo, principalmente cuando se produce variedad de productos, como vino tinto seco, tinto semiseco, abocado, borgoña, burdeos, blanco seco, blanco semiseco, rosé, dulces, etc.; además de otras características propias de desempeño de las empresas, en elaboración de vinos. El problema específico cuando existen varias líneas de producción, y se utiliza un método de costeo tradicional es la alteración en el costo de producción, debido que la herramienta de asignación que aplica este método es muy básica, no apropiada para la distribución de los costos indirectos; en consecuencia proporciona un costo impreciso, arbitrario y hasta homogéneo para todos los productos elaborados, independientemente de la variedad producida; con mucha frecuencia se presenta el subsidio cruzado de costos; es decir se imputa mayor costo a productos de elaboración simple, pero con mayor volumen de producción, y lo contrario; se asigna menor costo a productos de mayor complejidad, pero de menor volumen de producción. Este problema es muy común, no obstante, con tales deficiencias determinan costos de producción distorsionados, márgenes de ganancia ilusorios y precios aparentes; no ofrece una visión clara, en consecuencia, puede ocasionar pérdidas. De ahí se plantea ¿La implementación de un método de costeo basado en actividades incide en la fijación del precio en las empresas agroindustriales vitivinícolas de la Provincia de Tacna, 2017?

Un primer enfoque ayudará a mejorar las decisiones estratégicas en la fijación de precios; conocer la verdadera rentabilidad e incrementarla, así mismo disponer de información individualizada por tipo de vino; proporcionar un tratamiento eficiente de control y reducción de costos; gestionar la administración de recursos, y racionalizar actividades. Además, servir como referencia, porque suministra abundante información, provee de una visión clara de la asignación de costos con varias líneas de productos; e incrementa la productividad y desarrolla ventajas competitivas para las empresas productoras de vinos. El segundo enfoque, es de tipo social; debido que existe interés y respaldo por parte del gobierno peruano, en promover la imagen y marca del vino y pisco nacional, sumado a ello las acciones de instituciones encargadas de promover e impulsar a 
las empresas del sector manufacturero en la provincia de Tacna. Corresponde al ámbito del presente trabajo, y desde la contabilidad de gestión; fomentar con estudios relacionados, como la implementación de un procedimiento de gestión de costos innovador, que se ajuste a las necesidades del entorno empresarial; producir información especializada a nivel regional sobre empresas vitivinícolas como material de consulta en estudios similares; comprender e impulsar la industria del vino; y por último fortalecer la imagen del vino tacneño como bebida nacional por ser distintivo y contener la identidad de la provincia tacneña.

Contreras \& Mac Cawley (2006), desagregó y clasificó el proceso de elaboración del vino en actividades para la asignación el costo a través de inductores, en función a la utilización de los recursos por actividad más no por volumen de producción. El estudio es concluyente respecto del procedimiento en la implementación del $A B C$ al imputar los costos sobre el nivel de intensidad de las actividades, es decir permite una adecuada asignación de costos a las distintas líneas de producto, y en consecuencia obtener la verdadera rentabilidad porque identifica con qué categoría de vino se gana más. Möller (2011) menciona que el sistema de costeo basado en las actividades $(A B C)$. Implantación en una bodega, es determinar el costo del vino producido a través de las diversas actividades que implica producirlo. Los resultados obtenidos fueron, la determinación real del margen de rentabilidad para cada marca o variedad, precisar el precio con el que puede ingresar a un nuevo mercado, el punto de equilibrio en las ventas; así también los componentes en el costo total del vino, la proporción de las actividades en el costo lo que a la vez permite establecer que actividad dentro del proceso productivo le genera más costo, y también la identificación y estimación de actividades auxiliares que suelen pasar inadvertidas pero son importantes en la elaboración del producto final. Martínez B. \& Benitez B. (2013) dice que diseño implementado establece claramente qué tipo de actividades principales y de apoyo comprende el procesamiento y cuáles generan mayor costo, como fue el empacado en dos de sus tipos de vinos, así mismo la identificación costos indirectos elevados lo que conlleva a una gestión ineficiente, por estas razones la aplicación del sistema de costeo basado en actividades permite una visión clara sobre los costos incurridos, gestionarlos y llevar un adecuado control, por lo tanto conocer el precio con mayor exactitud, lo que conlleva a disponer de un margen de utilidad real.

Don \& Milordo (Don \& Milordo, 2010) con el propósito de determinar los costos, mediante la implementación de costos basados en actividades que, a través de su aplicación permite crear un entorno apropiado para obtener una relación de causa - efecto con mayor precisión entre factores, actividades y costos. Los resultados de esta metodología proporcionaron mayor precisión, porque el costo obtenido fue por variedad de uva en función a la producción por hectárea; se logró identificar claramente la existencia de costos superiores en dos de las cinco variedades, así también esta metodología es efectiva para asignar los costos indirectos en productos y servicios utilizados, mejora su control, aporta mayor información, al identificar las actividades que crean valor y aquellas que no, en consecuencia es factible adoptar decisiones más acertadas como reducir o eliminar actividades que se muestran menos efectivas para la empresa. Abanto \& Romero (2015) implementó un sistema de costeo ABC, para el proceso productivo de cerveza, y determinó la rentabilidad por producto, mediante el análisis de procesos, actividades y tareas; los resultados de la implementación del método $A B C$ reflejaron, una asignación de costos indirectos más realista, con una variación diferenciada de costos para los cuatro productos en ambos métodos, tradicional y $A B C$, donde el producto más económico según la empresa, resultó ser el más costoso, es decir se encontraba subsidiado, sin embargo el producto que absorbía gran parte de los costos indirectos, resultó ser más económico, es decir se encontraba sobre costeado. Y con respecto a las utilidades por producto, reflejan resultados más certeros sobre cuál es el más rentable. Celis Melendez (2009). buscó determinar la mejora en la eficiencia de una empresa dedicada a la producción de conservas de espárrago. Los resultados determinan que tanto las 
actividades principales como secundarias para cada proceso, otorgan mayor precisión al obtener el costo por hectárea, kilogramo y hora hombre respecto de las labores agrícolas implicadas, así también para las actividades agroindustriales, los costos unitarios para cada una de sus cuatro formas de presentación, lo que permite identificar y comparar cómo se utilizaron los recursos y en consecuencia las utilidades generadas. Todos estos resultados concluyen que el método de costeo $A B C$ ofrece una correcta aplicación de los costos indirectos que afectan los procesos y productos, convirtiéndose en herramienta de gestión muy útil y apropiada porque aporta información interna detallada que permite tener una visión más real sobre los costos y desempeño empresarial. Alfaro \& León (2015) aplicó el sistema de costos ABC para la fijación de precios en las empresas fabricantes de harina de maca, obtuvo un costo unitario más exacto, comparado con el costo regular mediante costeo tradicional, el resultado demostró que el sistema de costeo $A B C$ mejora la fijación de precios, debido a que lleva un control más específico por elemento del costo, puntualizando en el tratamiento de los costos indirectos de fabricación; por ello es herramienta de gestión, su implementación proporciona costos efectivos y toma de decisiones estratégicas.

Se decidió determinar si la implementación de un método de costeo basado en actividades incide en la fijación del precio en las empresas agroindustriales vitivinícolas de la provincia de Tacna, analizar si la asignación de costos indirectos en las actividades de producción en las empresas agroindustriales vitivinícolas incide en la fijación del precio, establecer si la implementación de un método de costeo basado en actividades, afecta al costo de producción y analizar si la implementación de un método de costeo basado en actividades incide en el margen de ganancia en las empresas agroindustriales vitivinícolas de la provincia de Tacna, 2017.

\section{Metodología de Costeo Basado en Actividades}

Esta metodología de costeo se enfoca en el tratamiento de los costos indirectos, los que no se asignan a los productos o servicios sino a las actividades que se realizan para producirlos. (Vaca López, 2012, pág. 9). El profesor Robert S. Kaplan de la Harvard Bussines School, propone la metodología $A B C$, fundamentada en la hipótesis; que son las actividades las que consumen recursos y originan los costos, no los productos, éstos solo requieren actividades necesarias para obtenerlos. El costeo basado en actividades $(A B C)$ es una metodología que cuantifica los costos a través de las actividades desarrolladas, recursos utilizados y objetos de costo. Este método asigna primero los recursos a las actividades, posteriormente, los costos de aquellas actividades se cargan a los objetos de costo (Cuevas, 2012, pág. 352). Facilita la identificación y disminución de costos inclusive posibles costos ocultos a través del nivel de análisis específico que proporciona. Es una herramienta útil para pequeñas y medianas empresas porque ofrece sencillez y claridad en su metodología de trabajo, tiene capacidad para reemplazar documentos contables engorrosos por información efectiva, tiene una perspectiva que permite comprender el desempeño real de una empresa. (Perucontable, 2017)

Cuervo \& Osorio, (2013) señalan que la metodología, es uno de los caminos para implementar el sistema de costeo $A B C$, los pasos propuestos son:

Paso 1: Información preliminar: Debe iniciar con un documento que deberá incluir, información sobre el grupo de trabajo, justificación del proyecto, objetivo general y específicos, metodología, alcance del trabajo, recursos, duración y cronograma.(Cuervo \& Osorio, 2013).

Paso 2: Establecimiento del proyecto: Se establece el plan de trabajo (acciones necesarias, objetivos de implementación, cronograma de trabajo, capacitación de personal, sensibilización, concientización) y 
ser parte de la premisa que la cultura del costo es una característica necesaria para que la empresa logre sus propósitos y perdure (Cuervo \& Osorio, 2013).

Paso 3: Diagnóstico de la contabilidad: Revisión de los sistemas de contabilidad fuente principal del modelo ABC. El diseño de una contabilidad por áreas de responsabilidad, debe quedar documentado, a través de un manual de procedimientos para evitar cualquier improvisación; la empresa de manera individualizada adopta la forma de distribución de costos y gastos que son indirectos, finalmente disponer de un inventario actualizado de propiedad, planta y equipo, clasificado por áreas de responsabilidad (Cuervo \& Osorio, 2013).

Paso 4: Identificación y definición de los objetos de costeo: El objeto de costo, es todo aquello que la empresa desea costear, como un cliente, un proveedor, un producto o servicio, máquinas, personal, puntos de venta, etc. (Cuervo \& Osorio, 2013).

Paso 5: Diseño de la estructura de navegación del modelo ABC: consiste en elaborar un mapa de costos que refleje de manera integral y gráfica, el desarrollo del sistema de costeo bajo la metodología $A B C$, a partir del consumo de recursos hasta el cálculo de los costos, además incluir las áreas de responsabilidad, actividades y los objetos de costo, agrupados por procesos, mediante la utilización de señas, marcas, colores, flechas, que establezca relación entre estos elementos. (Cuervo \& Osorio, 2013).

Paso 6: Diseño del diccionario de procesos y actividades: Éste contiene una compilación de las actividades desarrolladas por la entidad. Todas las actividades que salen en el diccionario se deben costear. No se puede permitir que tareas insignificantes se infiltren en el diccionario; tener una visión estratégica de éstas permite concentrarse en lo mayúsculo y maximiza esfuerzos de mejora continua. La metodología para desarrollar el diccionario, es a través de la carta organizacional y el listado de centros de costos, a fin de crear una ruta de las áreas que se visitarán en el tiempo previsto, deberá comunicarse el objetivo de la reunión, los propósitos y beneficios del modelo ABC (Cuervo \& Osorio, 2013).

Paso 7: Obtención de información sobre las actividades: Luego de tener el diccionario de actividades documentado y aprobado, se inicia con el diseño de formatos, con el propósito que cada empleado obtenga información sobre las actividades que realiza; la otra forma de recoger información es a través de estudios de tiempos y movimientos o del análisis de las fichas técnicas de costos de cada producto o servicio. A cada empleado se le entregará una encuesta, es conveniente, tener al alcance el diccionario de actividades y el listado de recursos. (Cuervo \& Osorio, 2013).

Paso 8: Identificación de los recursos consumidos: Se debe evaluar los recursos operacionales, esto incluye actividades operativas y administrativas; este modelo se enfoca en los recursos indirectos (Cuervo \& Osorio, 2013).

Paso 9: Definición de direccionadores o conductores: Para la selección de un direccionador, conductor, inductor o driver se debe tener en cuenta, la relación de causalidad, independencia del inductor, ser económico y fácil de usar, oportunidad, consistencia, y control constante con la finalidad de determinar variaciones no justificadas en éste. (Cuervo \& Osorio, 2013).

Paso 10: Tabulación de la información: Toda la información, deberá procesarse en el software de costos.

Paso 11: Cálculo de los costos unitarios: es necesario contar con una información de costos unitarios, que son un buen punto de partida para asegurar una evaluación razonable, analítica de los objetos de costos, actividades o productos finales. (Cuervo \& Osorio, 2013).

Paso 12: Implementación y seguimiento del modelo: la implementación es el reconocimiento formal del modelo de costos como un subsistema dentro de la entidad. Referente al seguimiento del modelo, implica: informe de costos de recursos, de áreas de responsabilidad, de actividades, procesos, objetos de costos; asignación de responsabilidad; evaluación de desempeño, en resumen cuantificar el trabajo que cada personal desempeña para lograr las metas en términos de costos. (Cuervo \& Osorio, 2013). 


\section{El Vino}

Para la Organización Internacional de la Viña y el Vino (OIV) (2017), "el vino es, exclusivamente, la bebida resultante de la fermentación alcohólica, completa o parcial, de uvas frescas, estrujadas o no, o de mosto de uva. Su contenido en alcohol adquirido no puede ser inferior a 8,5\% vol.", también la OIV, señala que, dependiendo de las características propias de las regiones como las condiciones del clima, suelo, la variedad, o las tradiciones, el grado alcohólico total mínimo podrá ser $7 \%$ vol., además de otras características técnicas que debe cumplir para ser considerado como vino. Existe gran variedad de vinos, que pueden clasificarse en función a sus características. El Instituto Nacional de Defensa de la Competencia y de la Protección de la Propiedad Intelectual INDECOPI, señala que los vinos se pueden clasificar:

- Por su color: Vinos tintos, blancos, rosados (INDECOPI, 2011)

- Por su contenido de azúcares reductores: a) Seco: Cuando el vino tiene un contenido máximo de $4 \mathrm{~g} / \mathrm{L}$ de azúcar. b) Semiseco: este tiene un contenido de azúcar más elevado que el vino seco, pero hasta un máximo de $90 \mathrm{~g} / \mathrm{L}$. y c) Dulce: este tipo de vino tiene un contenido de azúcar superior a $90 \mathrm{~g} / \mathrm{L}$. (INDECOPI, 2011)

- Por la técnica de elaboración: Vinos Especiales éstos trasladan características del tipo de uva, y también de la técnica aplicada en su producción. Incluye los licorosos y espumantes. (INDECOPI, 2011)

- Por crianza: Vinos criados en madera (Vino Gran Reserva, Vino Reserva, Vino Crianza), Vinos criados sin madera (tintos, blancos o rosados, pero criados sin presencia de madera) y vino Joven (Este vino se produce para comercializarse en forma inmediata, puede contener o no, vinos criados en madera de roble) (INDECOPI, 2011).

El cultivo de vid en el país, constituye una actividad productiva de gran importancia. A nivel nacional las zonas productoras de uva, se encuentran ubicadas principalmente en la costa sur: Lima, Ica, Arequipa, Moquegua y Tacna; así también en parte de la costa norte como La Libertad. (Ministerio de Agricultura y Riego, 2008). Cabe mencionar que las zonas productoras de uva antes mencionadas comprenden todo tipo de variedad de uva en su producción. Si bien las condiciones de clima y suelo son particulares en cada una de las regiones costeras productoras de uva vinífera; la provincia de Tacna tiene sus propias variedades. La producción de uva en la región de Tacna, que incluye las provincias de Tacna y Jorge Basadre; en donde destacan dos variedades: uva Italia con una proporción del $56 \%$ del total de hectáreas cultivadas, y la uva Negra criolla con el $29 \%$ del total de hectáreas destinadas para su cultivo, en relación a la superficie total para todas las variedades. Ambas poseen características distintivas, son las cepas por excelencia en el sur del país y las más representativas en la provincia de Tacna.

\section{METODOLOGÍA}

Las variables estudiadas son: Método de costeo basado en actividades (Indicadores: Actividades de producción), Fijación del precio (Indicadores: Costo de producción y margen de ganancia) y como variables Interviniente los niveles de demanda que teóricamente afectan a la variable dependiente. El tipo de investigación fue aplicada, porque pone en práctica las teorías del método de costeo basado en actividades para determinar el costo de la producción y la fijación del precio en las empresas agroindustriales vitivinícolas de la provincia de Tacna para el período de 2017. El diseño de investigación se limita a observar los acontecimientos sin intervenir en los mismos; mide variables o conceptos con el fin de especificar características de los procesos de costeo utilizados en las empresas agroindustriales vitivinícolas de la provincia de Tacna; además el 
estudio desarrollado usa el análisis transversal, debido que se recolectó información correspondiente al período 2017.

Ámbito y tiempo social de la investigación, provincia de Tacna. La unidad de estudio fueron las empresas agroindustriales vitivinícolas. La población en estudio es finita, conformada por 22 empresas agroindustriales vitivinícolas de la provincia de Tacna, correspondiente al padrón manufacturero industrial de la Dirección Regional de la Producción - Tacna. Muestra: Hernández citado en Castro (2003), expresa que "si la población es menor a cincuenta (50) individuos, la población es igual a la muestra" (pág.69).

El procedimiento de recopilación de la información consistió en acudir a las entidades como la Dirección Regional de la Producción, Dirección Regional de Agricultura, Municipalidad Provincial de Tacna, como fuentes para la recolección de información con el alcance necesario; así también se recopiló de trabajos de investigación, libros, revistas, periódicos y documentos similares. Técnicas del Análisis documental. La aplicación de esta técnica permitió analizar la documentación, actividades de producción, proceso productivo, materia prima utilizada, mano de obra especializada y los costos indirectos de fabricación fijos y variables.

Para el procesamiento de la información se utilizó los siguientes instrumentos: Análisis de contenido. Se procedió a la preparación de fichas de análisis de contenido, las cuales fueron debidamente elaboradas, ordenadas y numeradas, conteniendo información recopilada de las empresas agroindustriales vitivinícolas de la provincia de Tacna, 2017.

\section{RESULTADOS}

Tabla 1

Comparación de los costos de producción entre el método de costeo $A B C$ y método tradicional por órdenes de trabajo (O.T.), 2017.

\begin{tabular}{|c|c|c|c|c|c|c|}
\hline \multirow{3}{*}{ Empresa } & \multicolumn{6}{|c|}{ Costo de Producción } \\
\hline & \multicolumn{3}{|c|}{$\mathrm{ABC}$} & \multicolumn{3}{|c|}{ Tradicional } \\
\hline & $\begin{array}{c}\text { O.T / } \\
\text { Tinto } \\
\text { Semiseco }\end{array}$ & $\begin{array}{c}\text { O.T / } \\
\text { Abocado }\end{array}$ & $\begin{array}{c}\text { O.T / } \\
\text { Blanco } \\
\text { Semiseco } \\
\end{array}$ & $\begin{array}{c}\text { O.T / } \\
\text { Tinto } \\
\text { Semiseco }\end{array}$ & $\begin{array}{c}\text { O.T / } \\
\text { Abocado }\end{array}$ & $\begin{array}{c}\text { O.T / } \\
\text { Blanco } \\
\text { Semiseco }\end{array}$ \\
\hline$E-1$ & S/ 9.752 & S/ 9.950 & S/ 7.227 & S/ 10.554 & S/ 10.936 & $\mathrm{~S} / 10.018$ \\
\hline$E-2$ & S/ 9.934 & S/ 9.987 & S/ 6.234 & S/ 11.060 & S/ 11.039 & S/ 7.824 \\
\hline$E-3$ & S/ 10.503 & S/ 11.064 & S/ 9.174 & S/ 11.537 & S/ 12.421 & S/ 11.529 \\
\hline$E-4$ & S/ 9.815 & S/ 10.057 & S/ 5.922 & S/ 10.724 & S/ 11.371 & S/ 7.224 \\
\hline$E-5$ & S/ 10.593 & S/ 11.505 & S/ 8.092 & S/ 11.750 & S/ 13.416 & S/ 9.792 \\
\hline$E-6$ & S/ 9.872 & S/ 9.985 & S/ 7.214 & S/ 10.837 & S/ 11.045 & S/ 10.513 \\
\hline$E-7$ & S/ 9.270 & S/ 9.375 & S/ 6.726 & S/ 10.277 & S/ 10.611 & S/ 10.650 \\
\hline$E-8$ & S/ 9.829 & S/ 10.192 & S/ 6.293 & S/ 10.652 & S/ 11.568 & S/ 7.912 \\
\hline$E-9$ & S/ 10.488 & S/ 11.549 & S/ 13.981 & S/ 11.354 & S/ 13.058 & S/ 18.330 \\
\hline$E-10$ & S/ 9.269 & S/ 9.292 & S/ 6.831 & S/ 10.392 & S/ 10.557 & S/ 11.382 \\
\hline $\mathrm{E}-11$ & S/ 9.949 & $S / 10.113$ & S/ 6.169 & S/ 11.136 & S/ 11.609 & S/ 7.894 \\
\hline $\mathrm{E}-12$ & S/ 10.330 & S/ 11.545 & S/ 13.065 & S/ 11.218 & S/ 13.225 & S/ 15.772 \\
\hline $\mathrm{E}-13$ & S/ 10.824 & S/ 11.555 & S/ 13.586 & S/ 12.063 & S/ 13.329 & S/ 16.653 \\
\hline E-14 & S/ 9.944 & S/ 10.039 & S/ 5.969 & S/ 11.112 & S/ 11.213 & S/ 7.276 \\
\hline E-15 & S/ 9.965 & S/ 9.985 & S/ 6.340 & S/ 11.084 & S/ 11.118 & S/ 8.186 \\
\hline$E-16$ & S/ 9.962 & S/ 9.988 & S/ 6.599 & S/ 11.156 & S/ 11.227 & S/ 8.772 \\
\hline E-17 & S/ 10.547 & S/ 12.035 & S/ 19.025 & S/ 11.636 & S/ 14.241 & S/ 26.343 \\
\hline E-18 & S/ 11.192 & S/ 11.942 & S/ 10.905 & S/ 12.468 & S/ 13.516 & S/ 12.743 \\
\hline E-19 & S/ 10.104 & S/ 9.914 & S/ 6.360 & S/ 11.450 & S/ 10.921 & S/ 8.069 \\
\hline$E-20$ & S/ 9.893 & S/ 10.214 & S/ 6.075 & S/ 10.862 & S/ 11.556 & S/ 7.624 \\
\hline$E-21$ & S/ 9.978 & S/ 10.076 & S/ 6.878 & S/ 11.141 & S/ 11.301 & S/ 9.223 \\
\hline E-22 & S/ 10.865 & S/ 11.002 & S/ 6.654 & S/ 11.710 & S/ 12.001 & S/ 8.539 \\
\hline
\end{tabular}

Tabla 2

Comparación de los márgenes de ganancia entre el método de costeo $A B C$ y el método tradicional por órdenes de trabajo (O.T.), 2017.

\begin{tabular}{|c|c|c|c|c|c|c|}
\hline \multirow[t]{3}{*}{ Empresa } & \multicolumn{6}{|c|}{ Margen de ganancia } \\
\hline & \multicolumn{3}{|c|}{$\mathrm{ABC}$} & \multicolumn{3}{|c|}{$\begin{array}{r}\text { Tradicional } \\
\end{array}$} \\
\hline & $\begin{array}{c}\text { O.T / } \\
\text { Tinto } \\
\text { Semiseco } \\
\end{array}$ & $\begin{array}{c}\text { O.T / } \\
\text { Abocado }\end{array}$ & $\begin{array}{c}\text { O.T / } \\
\text { Blanco } \\
\text { Semiseco } \\
\end{array}$ & $\begin{array}{c}\text { O.T / } \\
\text { Tinto } \\
\text { Semiseco } \\
\end{array}$ & $\begin{array}{c}\text { O.T / } \\
\text { Abocado }\end{array}$ & $\begin{array}{c}\text { O.T / } \\
\text { Blanco } \\
\text { Semiseco } \\
\end{array}$ \\
\hline $\mathrm{E}-1$ & $41.02 \%$ & $43.22 \%$ & $55.34 \%$ & $37.90 \%$ & $39.32 \%$ & $39.93 \%$ \\
\hline $\mathrm{E}-2$ & $35.23 \%$ & $35.05 \%$ & $40.10 \%$ & $31.65 \%$ & $29.89 \%$ & $31.95 \%$ \\
\hline$E-3$ & $21.90 \%$ & $21.69 \%$ & $25.07 \%$ & $19.94 \%$ & $19.32 \%$ & $19.95 \%$ \\
\hline$E-4$ & $27.51 \%$ & $27.84 \%$ & $33.77 \%$ & $25.18 \%$ & $24.62 \%$ & $27.68 \%$ \\
\hline$E-5$ & $21.71 \%$ & $23.47 \%$ & $27.19 \%$ & $19.57 \%$ & $20.13 \%$ & $22.47 \%$ \\
\hline$E-6$ & $27.35 \%$ & $28.04 \%$ & $36.04 \%$ & $24.91 \%$ & $25.35 \%$ & $24.73 \%$ \\
\hline$E-7$ & $24.81 \%$ & $24.53 \%$ & $34.20 \%$ & $22.38 \%$ & $21.68 \%$ & $21.60 \%$ \\
\hline $\mathrm{E}-8$ & $26.45 \%$ & $27.47 \%$ & $33.37 \%$ & $24.41 \%$ & $24.20 \%$ & $26.54 \%$ \\
\hline$E-9$ & $25.74 \%$ & $22.51 \%$ & $25.03 \%$ & $23.78 \%$ & $19.91 \%$ & $19.09 \%$ \\
\hline$E-10$ & $13.41 \%$ & $13.56 \%$ & $10.77 \%$ & $14.74 \%$ & $15.05 \%$ & $16.14 \%$ \\
\hline $\mathrm{E}-11$ & $28.14 \%$ & $28.68 \%$ & $37.28 \%$ & $25.14 \%$ & $24.98 \%$ & $29.14 \%$ \\
\hline $\mathrm{E}-12$ & $22.26 \%$ & $22.52 \%$ & $23.73 \%$ & $20.50 \%$ & $19.66 \%$ & $19.66 \%$ \\
\hline$E-13$ & $22.17 \%$ & $23.37 \%$ & $22.08 \%$ & $19.90 \%$ & $20.26 \%$ & $18.01 \%$ \\
\hline E-14 & $28.16 \%$ & $27.89 \%$ & $33.51 \%$ & $25.20 \%$ & $24.97 \%$ & $27.49 \%$ \\
\hline E-15 & $34.12 \%$ & $33.05 \%$ & $37.86 \%$ & $30.68 \%$ & $29.68 \%$ & $29.32 \%$ \\
\hline E-16 & $35.13 \%$ & $40.05 \%$ & $48.49 \%$ & $31.37 \%$ & $35.63 \%$ & $36.48 \%$ \\
\hline E-17 & $21.81 \%$ & $23.27 \%$ & $23.13 \%$ & $19.77 \%$ & $19.66 \%$ & $16.70 \%$ \\
\hline E-18 & $22.34 \%$ & $22.61 \%$ & $22.01 \%$ & $20.05 \%$ & $19.98 \%$ & $18.83 \%$ \\
\hline E-19 & $44.54 \%$ & $43.37 \%$ & $62.89 \%$ & $39.30 \%$ & $39.37 \%$ & $49.57 \%$ \\
\hline$E-20$ & $27.29 \%$ & $27.41 \%$ & $32.92 \%$ & $24.86 \%$ & $24.23 \%$ & $26.23 \%$ \\
\hline$E-21$ & $27.06 \%$ & $27.79 \%$ & $33.44 \%$ & $24.23 \%$ & $24.78 \%$ & $24.94 \%$ \\
\hline $\mathrm{E}-22$ & $26.69 \%$ & $27.27 \%$ & $33.06 \%$ & $24.76 \%$ & $25.00 \%$ & $25.76 \%$ \\
\hline
\end{tabular}

Fuente: Basado en información contable $\mathrm{y}$ operativa de las empresas agroindustriales vitivinícolas, provincia de Tacna, 2017. 
Tabla 3

Comparación de precios entre el método de costeo $A B C$ y el método tradicional por órdenes de trabajo (O.T.), 2017.

\begin{tabular}{|c|c|c|c|c|c|c|}
\hline \multirow[b]{3}{*}{ Empresa } & \multicolumn{6}{|c|}{ Precios } \\
\hline & \multicolumn{3}{|c|}{ ABC } & \multicolumn{3}{|c|}{ Tradicional } \\
\hline & $\begin{array}{c}\text { O.T / } \\
\text { Tinto } \\
\text { Semiseco }\end{array}$ & $\begin{array}{c}\text { O.T / } \\
\text { Abocado }\end{array}$ & $\begin{array}{c}\text { O.T / } \\
\text { Blanco } \\
\text { Semiseco }\end{array}$ & $\begin{array}{c}\text { O.T / } \\
\text { Tinto } \\
\text { Semiseco }\end{array}$ & $\begin{array}{c}\text { O.T / } \\
\text { Abocado }\end{array}$ & $\begin{array}{c}\text { O.T / } \\
\text { Blanco } \\
\text { Semiseco }\end{array}$ \\
\hline $\mathrm{E}-1$ & S/ 16.227 & S/ 16.815 & S/ 13.248 & S/ 17.174 & S/ 17.978 & S/ 16.542 \\
\hline$E-2$ & S/ 15.852 & S/ 15. & S/ 10.306 & 7.180 & & 2.182 \\
\hline$E-3$ & 15.108 & S/ 15.8 & S/ 13.540 & 16.328 & S/ 17. & \\
\hline$E-4$ & S/ 14.767 & S/ 15.171 & S/ 9.349 & 15.840 & & \\
\hline$E-5$ & S/ 15.214 & S/ 16.762 & S/ 12.145 & S/ 16.579 & S/ 19. & S/ 14.150 \\
\hline E-6 & S/ 14.835 & S/ 15.086 & S/ 11.581 & 5.974 & S/ 1 & $S / 15$ \\
\hline$E-7$ & S/ 13.653 & S/ 13.776 & S/ 10.650 & 841 & $S / 1$ & $S / 1$ \\
\hline E-8 & S/ 14.666 & S/ 15. & S/ 9.904 & 638 & S/ 1 & S/ 1 \\
\hline E-9 & 15 & S/ 16 & S/ 20.627 & & $S / 1$ & $S / 2$ \\
\hline$E-10$ & S/ 13.415 & S/ 13 & S/ 10.774 & & $S / 1$ & S/ 1 \\
\hline $\mathrm{E}-11$ & S/ 15.044 & S/ 15. & S/ 9.994 & & $S / 1$ & $S / 12$ \\
\hline $\mathrm{E}-12$ & S/ 14.904 & S/ 16.691 & S/ 19.074 & & S/ 18 & .269 \\
\hline$E-13$ & S/ 15.604 & S/ 16.820 & S/ 19.572 & S/ 17.066 & S/ 18.914 & S/ 23.191 \\
\hline$E-14$ & S/ 15.038 & S/ 15.150 & S/ 9.403 & & S/ 1 & s/ 1 \\
\hline E-15 & S/ 15.771 & S/ 15.676 & S/ 10.313 & S/ 17.091 & S/ 17. & S/ 12.491 \\
\hline$E-16$ & S/ 15.885 & S/ 16.505 & S/ 11.563 & S/ 1 & S/ 17 & S/ 14.127 \\
\hline $\mathrm{E}-17$ & S/ 15.159 & S/ 17.505 & S/ 27.642 & S/ 16.445 & S/ 20.108 & S/ 36.277 \\
\hline & S/ 16.156 & S/ 17.278 & S/ 15.700 & S/ 17.662 & S/ 19.135 & S/ 17.869 \\
\hline$E-19$ & S/ 17.232 & S/ 16.773 & S/ 12.225 & S/ 18.821 & S/ 17.961 & S/ 14.241 \\
\hline$E-20$ & S/ 14.859 & S/ 15.356 & S/ 9.528 & S/ 16.003 & S/ 16.940 & S/ 11.356 \\
\hline & S/ 14.959 & S/ 15.193 & S/ 10.830 & S/ 16.332 & S/ 16.639 & S/ 13.597 \\
\hline$E-22$ & S/ 16.242 & S/ 16.522 & S/ 10.447 & S/ 17.240 & S/ 17.701 & S/ 12.672 \\
\hline
\end{tabular}

Fuente: Basado en información contable y operativa de las empresas agroindustriales vitivinícolas, provincia de Tacna, 2017.

\section{Tabla 41}

Comparación de la asignación de costos indirectos de fabricación (CIF) entre el método de costeo $A B C$ y el método tradicional por órdenes de trabajo (O.T.), 2017.

\begin{tabular}{|c|c|c|c|c|c|c|c|c|}
\hline \multirow[b]{3}{*}{ Empresa } & \multicolumn{8}{|c|}{ Asignación de costos (CIF) } \\
\hline & \multicolumn{5}{|c|}{$\mathbf{A B C}$} & \multicolumn{3}{|c|}{ Tradicional } \\
\hline & & $\begin{array}{l}\text { T / } \\
\text { into } \\
\text { iseco }\end{array}$ & $\begin{array}{c}\text { O.T / } \\
\text { Abocado }\end{array}$ & & $\begin{array}{l}. \mathrm{T} / \\
\text { lanco } \\
\text { iseco }\end{array}$ & $\begin{array}{c}\text { O.T / } \\
\text { Tinto } \\
\text { Semiseco }\end{array}$ & $\begin{array}{c}\text { O.T / } \\
\text { Abocado }\end{array}$ & $\begin{array}{c}\text { O.T / } \\
\text { Blanco } \\
\text { Semiseco }\end{array}$ \\
\hline $\mathrm{E}-1$ & $\mathrm{~s} /$ & 0.296 & S/ 0.348 & S/ & 0.640 & $\mathrm{~S} / 1.098$ & S/ 1.334 & S/ 3.431 \\
\hline$E-2$ & S/ & 0.317 & S/ 0.375 & S/ & 0.514 & S/ 1.443 & S/ 1.428 & 2.104 \\
\hline$E-3$ & S/ & 0.392 & S/ 0.453 & s/ & 0.936 & S/ 1.426 & S/ 1.810 & 3.291 \\
\hline E-4 & S/ & 0.332 & S/ 0.341 & s/ & 0.436 & S/ 1.241 & S/ 1.655 & 1.738 \\
\hline E-5 & S/ & 0.465 & S/ 0.493 & S/ & 1.005 & S/ 1.622 & S/ 2.404 & S/ 2.704 \\
\hline$E-6$ & S/ & 0.324 & S/ 0.358 & S/ & 0.482 & S/ 1.289 & $\mathrm{~S} / 1.418$ & S/ 3.782 \\
\hline$E-7$ & S/ & 0.321 & S/ 0.312 & S/ & 0.499 & S/ 1.327 & S/ 1.548 & S/ 4.424 \\
\hline$E-8$ & S/ & 0.337 & S/ 0.354 & S/ & 0.501 & S/ 1.161 & S/ 1.731 & S/ 2.120 \\
\hline E-9 & S/ & 0.403 & S/ 0.466 & S/ & 1.576 & S/ 1.270 & S/ 1.975 & S/ 5.925 \\
\hline $\mathrm{E}-10$ & S/ & 0.318 & S/ 0.288 & S/ & 0.496 & S/ 1.442 & S/ 1.553 & S/ 5.048 \\
\hline $\mathrm{E}-11$ & S/ & 0.335 & S/ 0.330 & S/ & 0.467 & S/ 1.522 & S/ 1.826 & S/ 2.191 \\
\hline $\mathrm{E}-12$ & S/ & 0.366 & S/ 0.431 & S/ & 2.307 & S/ 1.253 & S/ 2.111 & S/ 5.014 \\
\hline$E-13$ & S/ & 0.429 & S/ 0.451 & S/ & 2.496 & S/ 1.669 & S/ 2.225 & S/ 5.563 \\
\hline E-14 & S/ & 0.335 & S/ 0.392 & S/ & 0.482 & S/ 1.503 & S/ 1.566 & S/ 1.789 \\
\hline $\mathrm{E}-15$ & S/ & 0.349 & S/ 0.353 & S/ & 0.501 & S/ 1.467 & S/ 1.487 & S/ 2.348 \\
\hline E-16 & S/ & 0.345 & S/ 0.345 & S/ & 0.598 & S/ 1.539 & S/ 1.583 & S/ 2.771 \\
\hline & S/ & 0.431 & S/ 0.498 & s/ & 2.821 & S/ 1.521 & S/ 2.704 & S/ 10.139 \\
\hline $\mathrm{E}-18$ & S/ & 0.377 & S/ 0.493 & S/ & 1.606 & S/ 1.653 & S/ 2.067 & S/ 3.444 \\
\hline $\mathrm{E}-19$ & S/ & 0.360 & S/ 0.358 & S/ & 0.566 & S/ 1.706 & S/ 1.365 & S/ 2.275 \\
\hline$E-20$ & S/ & 0.328 & S/ 0.387 & s/ & 0.396 & S/ 1.297 & S/ 1.729 & S/ 1.945 \\
\hline $\mathrm{E}-21$ & S/ & 0.322 & S/ 0.360 & S/ & 0.627 & S/ 1.486 & S/ 1.585 & 2.972 \\
\hline E-22 & S/ & 0.338 & S/ 0.353 & S/ & 0.482 & S/ 1.184 & S/ 1.353 & S/ 2.368 \\
\hline
\end{tabular}

Fuente: Basado en información contable y operativa de las empresas agroindustriales vitivinícolas, provincia de Tacna, 2017.
Tabla 5

Comparación de los costos de producción entre el método de costeo $A B C$ y el método tradicional por órdenes de trabajo (O.T.), 2017.

\begin{tabular}{|c|c|c|c|c|c|c|}
\hline \multirow[b]{3}{*}{ Empresa } & \multicolumn{6}{|c|}{ Costo de Producción } \\
\hline & \multicolumn{3}{|c|}{$\mathbf{A B C}$} & \multicolumn{3}{|c|}{ Tradicional } \\
\hline & $\begin{array}{c}\text { O.T / } \\
\text { Tinto } \\
\text { Semiseco }\end{array}$ & $\begin{array}{c}\text { O.T / } \\
\text { Abocado }\end{array}$ & $\begin{array}{c}\text { O.T / } \\
\text { Blanco } \\
\text { Semiseco }\end{array}$ & $\begin{array}{c}\text { O.T / } \\
\text { Tinto } \\
\text { Semiseco }\end{array}$ & $\begin{array}{c}\text { O.T / } \\
\text { Abocado }\end{array}$ & $\begin{array}{c}\text { O.T / } \\
\text { Blanco } \\
\text { Semiseco }\end{array}$ \\
\hline $\mathrm{E}-1$ & S/ 9.752 & S/ 9.950 & S/ 7.227 & S/ 10.554 & S/ 10.936 & S/ 10.018 \\
\hline$E-2$ & 9.934 & S/ 9.987 & S/ 6.234 & .060 & S/ 11. & S/ 7.824 \\
\hline E-3 & S/ 10.503 & S/ 11.064 & S/ 9.174 & & & S/ 11.529 \\
\hline E-4 & S/ 9.815 & S/ 10.057 & S/ 5.922 & & 71 & S/ 7.224 \\
\hline $\mathrm{E}-5$ & S/ 10.593 & S/ 11.505 & S/ 8.092 & & S/ 13.416 & S/ 9.792 \\
\hline$E-6$ & S/ 9.872 & S/ 9.985 & S/ 7.214 & & $S / 1$ & S/ 10.513 \\
\hline$E-7$ & 9.270 & S/ 9.375 & S/ 6.726 & & & 0.650 \\
\hline$E-8$ & S/ 9.829 & S/ 10.192 & S/ 6.293 & & $S / 1$ & S/ 7.912 \\
\hline$E-9$ & S/ 10.488 & $S / 1$ & S/ 13.98 & & & S/ 18.330 \\
\hline E-1C & S/ 9.269 & S/ 9.292 & S/ 6.831 & & S/ 10. & S/ 11.382 \\
\hline E-11 & 9.949 & S/ 10.113 & S/ 6.169 & & S/ 11 & S/ 7.894 \\
\hline E-12 & S/ 10.330 & S/ 1 & S/ 13.06 & & $S / 1$ & S/ 15.772 \\
\hline$E-1$ & S/ 10.824 & S/ 11.555 & S/ 13.58 & S/ 1 & S/ 13.329 & S/ 16.653 \\
\hline E-14 & S/ 9.944 & S/ 10.039 & S/ 5.969 & S/ 1 & S/ 11.213 & S/ 7.276 \\
\hline E-15 & S/ 9.965 & S/ 9.985 & S/ 6.340 & S/ 11.084 & S/ 11.118 & S/ 8.186 \\
\hline E-1 & S/ 9.962 & S/ 9.988 & S/ 6.599 & & S/ 11.227 & S/ 8.772 \\
\hline $\mathrm{E}-17$ & S/ 10.547 & S/ 12.035 & S/ 19.02 & & S/ 14.241 & S/ 26.343 \\
\hline $\mathrm{E}-18$ & S/ 11.192 & S/ 11.942 & S/ 10.905 & S/ 12. & S/ 13.516 & S/ 12.743 \\
\hline E-19 & S/ 10.104 & S/ 9.914 & S/ 6.360 & S/ 11.450 & S/ 10.921 & S/ 8.069 \\
\hline & S/ 9.893 & S/ 10.214 & S/ 6.07 & S/ 1 & S/ 11.556 & S/ 7.624 \\
\hline & S/ 9.978 & S/ 10.076 & S/ 6.878 & & S/ 11.301 & S/ 9.223 \\
\hline$E-22$ & S/ 10.865 & S/ 11.002 & S/ 6.654 & S/ 11.710 & S/ 12.001 & S/ 8.539 \\
\hline
\end{tabular}

Fuente: Basado en información contable y operativa de las empresas agroindustriales vitivinícolas, provincia de Tacna, 2017.

\section{Tabla 6}

Comparación de los márgenes de ganancia entre el método de costeo $A B C$ y el método tradicional por órdenes de trabajo (O.T.), 2017.

\begin{tabular}{|c|c|c|c|c|c|c|}
\hline \multirow[t]{3}{*}{ Empresa } & \multicolumn{6}{|c|}{ Margen de ganancia } \\
\hline & \multicolumn{3}{|c|}{$\mathrm{ABC}$} & \multicolumn{3}{|c|}{ Tradicional } \\
\hline & $\begin{array}{c}\text { O.T / } \\
\text { Tinto } \\
\text { Semiseco }\end{array}$ & $\begin{array}{c}\text { O.T / } \\
\text { Abocado }\end{array}$ & $\begin{array}{c}\text { O.T / } \\
\text { Blanco } \\
\text { Semiseco }\end{array}$ & $\begin{array}{c}\text { O.T / } \\
\text { Tinto } \\
\text { Semiseco }\end{array}$ & $\begin{array}{c}\text { O.T / } \\
\text { Abocado }\end{array}$ & $\begin{array}{c}\text { O.T / } \\
\text { Blanco } \\
\text { Semiseco }\end{array}$ \\
\hline E-1 & $41.02 \%$ & $43.22 \%$ & $55.34 \%$ & $37.90 \%$ & $39.32 \%$ & $39.93 \%$ \\
\hline E-2 & $35.23 \%$ & $35.05 \%$ & $40.10 \%$ & $31.65 \%$ & $29.89 \%$ & $31.95 \%$ \\
\hline E-3 & $21.90 \%$ & $21.69 \%$ & $25.07 \%$ & $19.94 \%$ & $19.32 \%$ & $19.95 \%$ \\
\hline E-4 & $27.51 \%$ & $27.84 \%$ & $33.77 \%$ & $25.18 \%$ & $24.62 \%$ & $27.68 \%$ \\
\hline E-5 & $21.71 \%$ & $23.47 \%$ & $27.19 \%$ & $19.57 \%$ & $20.13 \%$ & $22.47 \%$ \\
\hline E-6 & $27.35 \%$ & $28.04 \%$ & $36.04 \%$ & $24.91 \%$ & $25.35 \%$ & $24.73 \%$ \\
\hline E-7 & $24.81 \%$ & $24.53 \%$ & $34.20 \%$ & $22.38 \%$ & $21.68 \%$ & $21.60 \%$ \\
\hline E-8 & $26.45 \%$ & $27.47 \%$ & $33.37 \%$ & $24.41 \%$ & $24.20 \%$ & $26.54 \%$ \\
\hline E-9 & $25.74 \%$ & $22.51 \%$ & $25.03 \%$ & $23.78 \%$ & $19.91 \%$ & $19.09 \%$ \\
\hline E-10 & $13.41 \%$ & $13.56 \%$ & $10.77 \%$ & $14.74 \%$ & $15.05 \%$ & $16.14 \%$ \\
\hline E-11 & $28.14 \%$ & $28.68 \%$ & $37.28 \%$ & $25.14 \%$ & $24.98 \%$ & $29.14 \%$ \\
\hline E-12 & $22.26 \%$ & $22.52 \%$ & $23.73 \%$ & $20.50 \%$ & $19.66 \%$ & $19.66 \%$ \\
\hline E-13 & $22.17 \%$ & $23.37 \%$ & $22.08 \%$ & $19.90 \%$ & $20.26 \%$ & $18.01 \%$ \\
\hline E-14 & $28.16 \%$ & $27.89 \%$ & $33.51 \%$ & $25.20 \%$ & $24.97 \%$ & $27.49 \%$ \\
\hline E-15 & $34.12 \%$ & $33.05 \%$ & $37.86 \%$ & $30.68 \%$ & $29.68 \%$ & $29.32 \%$ \\
\hline E-16 & $35.13 \%$ & $40.05 \%$ & $48.49 \%$ & $31.37 \%$ & $35.63 \%$ & $36.48 \%$ \\
\hline E-17 & $21.81 \%$ & $23.27 \%$ & $23.13 \%$ & $19.77 \%$ & $19.66 \%$ & $16.70 \%$ \\
\hline E-18 & $22.34 \%$ & $22.61 \%$ & $22.01 \%$ & $20.05 \%$ & $19.98 \%$ & $18.83 \%$ \\
\hline E-19 & $44.54 \%$ & $43.37 \%$ & $62.89 \%$ & $39.30 \%$ & $39.37 \%$ & $49.57 \%$ \\
\hline E-20 & $27.29 \%$ & $27.41 \%$ & $32.92 \%$ & $24.86 \%$ & $24.23 \%$ & $26.23 \%$ \\
\hline E-21 & $27.06 \%$ & $27.79 \%$ & $33.44 \%$ & $24.23 \%$ & $24.78 \%$ & $24.94 \%$ \\
\hline E-22 & $26.69 \%$ & $27.27 \%$ & $33.06 \%$ & $24.76 \%$ & $25.00 \%$ & $25.76 \%$ \\
\hline
\end{tabular}

Fuente: Basado en información contable y operativa de las empresas agroindustriales vitivinícolas, provincia de Tacna, 2017.

La implementación de un método de costeo basado en actividades incide en la fijación del precio en las empresas agroindustriales vitivinícolas de la provincia de Tacna, 2017. Dado que el p-valor (0.000) es menor que el nivel de significancia (0.05). Por lo tanto, se concluye que a un margen 
de error del 5\% que la implementación de un método de costeo basado en actividades incide en la fijación del precio en las empresas agroindustriales vitivinícolas de la provincia de Tacna, 2017. La asignación de costos indirectos en las actividades de producción en las empresas agroindustriales vitivinícolas de la provincia de Tacna 2017, inciden en la fijación del precio ( $p$ : 0.000). La implementación de un método de costeo basado en actividades afecta al costo de producción en las empresas agroindustriales vitivinícolas de la provincia de Tacna, 2017 (p:0.000). La implementación de un método de costeo basado en actividades incide en el margen de ganancia en las empresas agroindustriales vitivinícolas de la provincia de Tacna, 2017 (p: 0.001).

\section{DISCUSIÓN}

En relación a la asignación de costos indirectos de fabricación en las actividades de producción; se determinó estadísticamente que la asignación de costos indirectos en las actividades de producción, incide considerablemente en la fijación del precio. Las empresas agroindustriales vitivinícolas de la provincia de Tacna, utilizan el método de costeo tradicional que aplican una tasa de asignación inadecuada apoyada en el volumen de producción, como medida para calcular los costos indirectos, lo que ocasionan costos promedio y a la vez elevado.

Los resultados estadísticos con el método tradicional, se reflejan incrementados en las tres variedades: vino tinto semiseco, abocado y blanco semiseco; en todos los casos de estudio, pero acentuado en las empresas: E-7, E-9, E-10 y E-17, con valores de $\mathrm{S} / 4.4, \mathrm{~S} / 5.9, \mathrm{~S} / 5.0$ y $\mathrm{S} / 10.1$, mientras que con el método de costeo $A B C$ los costos indirectos fueron: $S / 0.4, S / 1.5, S / 0.4$ y $S / 2.8$. Las diferencias son notables, y señala que existe sobrecosto, lo que confirma la ineficiencia del método tradicional para asignar o aplicar los costos indirectos de fabricación; en contraste con el método $A B C$, que utiliza inductores para medir el nivel de intensidad o consumo por actividad, y en propicia una asignación individualizada de los costos indirectos, en función al tipo de vino producido. Como lo mencionó (Abanto Vasquez \& Romero Baltodano, 2015, pág. 72), en su trabajo de tesis, donde confirma que el método $A B C$, distribuye con exactitud los costos indirectos de fabricación, dado que utiliza cost drivers o inductores como medida en las actividades; sin embargo la determinación de inductores debe ser minuciosa, con el fin de elegir los más apropiados.

Con respecto al costo de producción; se determinó estadísticamente que la implementación de un método de costeo basado en actividades afecta notablemente al costo de producción. Las empresas agroindustriales vitivinícolas de la provincia de Tacna, emplean el método de costeo tradicional, que se base en el volumen de producción, lo que ocasiona el subsidio cruzado de costos, es decir no distribuye equitativamente, es ineficiente para medir la productividad, no es analítico, porque desconoce cómo se generan los costos.

Los resultados revelan diferencias contundentes, en especial con la variedad, vino blanco semiseco, con énfasis en las empresas: E-9, E-10, E-17, donde los costos de producción fueron: $\mathrm{S} / 18.3, \mathrm{~S} / 11.3$ y $\mathrm{S} / 26.3$, mientras que con el método $A B C$, fue: $\mathrm{S} / 13.9, \mathrm{~S} / 6.8$ y $\mathrm{S} / 19.0$, respectivamente; en consecuencia, se puede afirmar que el método tradicional no se adapta a las necesidades en el procesamiento de vinos, no tiene capacidad para detectar las tareas que generan valor, y lo más importante, que no atribuye equitativamente los costos para los tres tipos de vino, es deficiente y limitado. Como referencia (Contreras \& Mac Cawley, 2006, pág. 32), afirma que, el método de costeo tradicional distribuye los costos fijos a través de los centros de costos y luego transfiere hacia los productos o servicios, es decir traslada los costos a las líneas de productos en función del volumen, mas no, por el requerimiento del proceso mismo; el efecto producido es el subsidio cruzado de costos, dado que no atribuye el consumo real de recursos por tipo de producto. 
En relación al margen de ganancia; se determinó estadísticamente que, la implementación de un método de costeo basado en actividades incide de manera trascendental en el margen de ganancia. Las empresas agroindustriales vitivinícolas de la provincia de Tacna, no disponen de un adecuado sistema de información de costos como punto de partida para determinar precios y márgenes de ganancia, sólo poseen información poco apropiada y parcial sobre sus costos de producción, por lo tanto, un margen de ganancia casi estándar y además bajo, no ofrece una visión clara sobre la rentabilidad por tipo de vino.

Los resultados estadísticos reflejan diferencias importantes; presentan una tendencia a manera de promedio entre los tipos de vino; los resultados más sobresalientes se presentaron en la variedad de vino blanco semiseco, en las empresas: E-1, E-6, E-7, E-16, E-19, con márgenes de $39.9 \%, 24.7 \%, 21.6 \%, 36.4 \%, 49.5 \%$, sin embargo con el método de costeo basado en actividades se obtuvo: $55.3 \%, 36.0 \%, 34.2 \%, 48.4 \%$ y $62.8 \%$; en consecuencia se puede afirmar que el método tradicional es inefectivo y limitado, para suministrar información. (Contreras \& Mac Cawley, 2006, pág. 33), señala; que los resultados a través del método de costeo basado en actividades son más acertados, porque reconocen todos los recursos y componentes que conforman el costo, más aún en procesos complejos, proveen de ventaja competitiva porque identifica los productos más rentables y los que menos ganancias producen en la empresa; mientras que los sistemas tradicionales, no son compatibles con los procesos actuales, ni con las necesidades empresariales de estos tiempos.

Por los resultados obtenidos a través de la prueba estadística $U$ de Mann-Whitney, para muestras independientes; y lo antes referido, se determinó de manera concluyente que la implementación del método de costeo basado en actividades incide en la fijación del precio en las empresas agroindustriales vitivinícolas de la provincia de Tacna, 2017.

Los resultados confirmaron que utilizar un método de costeo tradicional como herramienta de costos es ineficiente, produce información limitada, inexacta y distorsionada; demostró que, el método de costeo basado en actividades, es una herramienta eficiente para gestionar costos, mediante el tratamiento que utiliza para asignar los costos indirectos de fabricación; tiene capacidad de agregar valor a la empresa, porque se enfoca en mejorar procesos, actividades, y el consumo de recursos, atribuye de manera equitativa los costos de producción especialmente cuando se tiene varias líneas de producción. Se pudo comprobar que existen diferencias sustanciales en cuanto a la fijación de precios; con el método tradicional, se reflejan significativamente altos en once empresas, las más resaltantes fueron: E-7, E-9, E-10 y E-17 cuyos precios resultaron: $S / 15, S / 25, S / 16$, y $S / 36$, en contraste con el método de costeo basado en actividades que fue de $S / 10, S / 20, S / 10$, y $S / 27$, respectivamente. Sin embargo, a pesar de todos los atributos que tiene este método de costeo, es fundamental disponer de información detallada y completa sobre la empresa y el proceso de producción antes de implementarlo, de lo contrario se puede incurrir en inexactitud. (Möller, 2011, pág. 78). Se concluye que implementar el método de costeo basado en actividades, es más beneficioso, porque el mecanismo de trabajo es muy adaptable al sector vitivinícola, proporciona cuantiosa información individualizada por tipo de producto, además permite mayor visibilidad y comprensión de las causas que ocasionan los costos.

De tal modo se concluye lo siguiente: que estadísticamente a un margen de error del $5 \%$, la asignación de los costos indirectos de fabricación en las actividades de producción, inciden de manera significativa en la fijación del precio en las empresas agroindustriales vitivinícolas de la provincia de Tacna, 2017. En cuanto a la evaluación de resultados, sobre la asignación de costos indirectos en las actividades de producción se puede demostrar mayor exactitud y objetividad al imputar los costos en las tareas relacionadas con la producción de vinos; este proceso conduce a 
la identificación de las actividades más costosas, como es el caso de la vendimia, recepción, selección, y a la vez, el embotellado; que en efecto generan mayor consumo de recursos y servicios para la producción en los tres tipos de vinos, pero en forma predominante en el vino blanco semiseco. Del mismo modo en relación al valor de los costos indirectos de fabricación, se puede percibir que el producto que predomina en costos indirectos más elevados es el vino blanco semiseco. Por lo tanto, el método de costeo basado en actividades, es eficiente para asignar costos y medir el consumo de recursos por actividades de producción; así también permite advertir el producto más oneroso en cuanto a sus costos indirectos; en consecuencia, la fijación de precios resulta más objetiva, por lo tanto, es posible evaluar y justificar el mantenimiento y/o permanencia de un producto en la empresa.

Se determinó estadísticamente a un margen de error del 5\%, que, la implementación de un método de costeo basado en actividades afecta significativamente al costo de producción en las empresas agroindustriales vitivinícolas de la provincia de Tacna, 2017. De la evaluación de resultados obtenida, en el tratamiento de los costos de producción, en las empresas agroindustriales vitivinícolas donde la producción de vinos es diversa; se puede comprobar que el método de costeo basado en actividades, es una herramienta idónea, dado que organiza a las empresas en un conjunto de actividades, esto permite establecer qué tareas intervienen efectivamente en la producción, y en consecuencia distribuir los costos. Es eficiente y específico al proporcionar una base correcta, puesto que los inductores facilitan la distribución del consumo de recursos por actividad; así mismo, resultó equitativo en la asignación, debido que personaliza los costos de producción incurridos realmente en el procesamiento por tipo de vino, de donde el vino blanco semiseco se destaca como el más costoso; y como el más económico, predomina el vino tinto semiseco. Por lo tanto, es una herramienta de gestión apropiada para establecer los costos de producción por tipo de producto.

Se determinó estadísticamente a un margen de error del 5\% que, la implementación de un método de costeo basado en actividades incide en el margen de ganancia en las empresas agroindustriales vitivinícolas de la provincia de Tacna, 2017. Respecto a la evaluación de resultados, en el margen de ganancia, se puede afirmar que el método de costeo basado en actividades, es una herramienta útil para tomar decisiones estratégicas y operativas, no solo por precisar con exactitud los costos de producción, sino por determinar la verdadera rentabilidad que posee cada tipo de vino. Mediante el método de costeo basado en actividades, se demostró superioridad en los indicadores de margen de ganancia en los tres productos, también se puede identificar el tipo de vino más rentable, donde destaca el vino blanco semiseco. Con el método tradicional los resultados se mostraron distorsionados a manera de bajo rendimiento, lo que representa una condición delicada que puede ocasionar efectos negativos al momento de fijar precios. Por lo tanto, el método de costeo basado en actividades es una herramienta estratégica integral que proporciona ventajas competitivas, al establecer indicadores de rentabilidad más exactos y al mismo tiempo precisión en la fijación de precios.

Se determinó estadísticamente a un margen de error del $5 \%$ que, la implementación de un método de costeo basado en actividades incide en la fijación del precio en las empresas agroindustriales vitivinícolas de la provincia de Tacna, 2017. De la evaluación de resultados obtenidos, a través de los indicadores analizados como los costos indirectos en las actividades de producción, el costo de producción y el margen de ganancia; se pudo demostrar que la aplicación del método de costeo basado en actividades, incide significativamente a través del tratamiento de estos indicadores en la fijación del precio. En efecto, reflejó valores más bajos, atractivos y reales así también diferenciados de acuerdo al tipo de vino: tinto semiseco, tinto abocado, y blanco semiseco. La industria del vino es un aspirante ideal para este método de costeo, debido a las características que posee como la diversidad en su producción y la competencia. Lo que debe 
despertar el interés, para efectuar una retroalimentación en sus procesos internos como un primer aspecto, pero relevante para incrementar su rendimiento económico y desempeño empresarial.

Así se recomienda a las empresas agroindustriales vitivinícolas de la provincia de Tacna, implementar medidas preliminares de mejora en las actividades de producción y en los costos indirectos de fabricación. Para tal efecto es necesario realizar un diagnóstico de los sistemas de contabilidad, revisión de las principales fuentes de información, inventario de activos, estructura de los centros de costos y principalmente del manual de procedimientos. En caso de no disponer de este documento, es esencial elaborarlo para establecer las actividades que intervienen en el proceso productivo, y clasificarlas. Posteriormente efectuar un análisis para el reconocimiento de todos los recursos y servicios directos o complementarios necesarios para el procesamiento de los diferentes tipos de productos.

\section{REFERENCIAS BIBLIOGRÁFICAS}

Abanto Vasquez, P. I., \& Romero Baltodano, P. I. (2015). Tesis. "Implementación de un sistema de costos $A B C$ para la determinación de la rentabilidad por producto en la empresa Corporación Libertad S.A.C. en Trujillo, 2015". Facultad de Negocios. Carrera de Contabilidad y Finanzas. Obtenido de http://repositorio.upn.edu.pe/handle/11537/9966

Alfaro, E., \& León, K. (11 de Mayo de 2015). Repositorio UPAO. Obtenido de http://repositorio.upa.edu.pe/handle/upaorep/1362

Castro, M. (2003). El Proyecto de Investigación y su Esquema de Elaboració. (2da. ed.). Caracas: Uyapal.

Celis Melendez, Y. M. (2009). Tesis de grado. "Método de costeo ABC para mejorar la eficiencia en una empresa agroindustrial de La Libertad". Universidad Nacional de Trujillo. Sección de Postgrado en Ciencias Económicas. 2009. Obtenido de http://dspace.unitru.edu.pe/handle/UNITRU/7667

Consejo-Normativo-de-Contabilidad. (23 de Agosto de 2017). Resolución № 003-2017-EF/30 Norma Internacional de Contabilidad № 2 Inventarios. Lima, Perú. Obtenido de https//:www.mef.gob.pe/es/consejo-normativo-de-contabilidad/niif

Consejo-Normativo-de-Contabilidad. (23 de Agosto de 2017). Resolución Nट 003-2017-EF/30. Obtenido de Ministerio de Economía y Finanzas: http://:mef.gob.pe/es/consejo-normatico-decontabilidad/niif

Contreras, H., \& Mac Cawley, A. (2006). Asociación de Economistas Agrarios A.G. Obtenido de http://www.aeachile.cl/wp-content/uploads/2017/07/Contreras-et-al.pdf

Cuervo, J., \& Osorio, J. (2013). Costeo Basado en actividades ABC: gestión basada en actividades ABC. Bogotá: Ecoe Ediciones. Obtenido de https://ebookcentral.proquest.com/lib/bibliotecauptsp/detail.action?doclD=4870521

Cuevas, C. (2012). Contabilidad de Costos (3ra. ed.). Pearson Educación. Obtenido de https://externalidadesintangibles.wordpress.com/2015/05/29/contabilidad-de-costos-3aedicion-2010-cuevas-carlos/

D.L.-№-1269. (17 de Diciembre de 2016). Decreto Legislativo que crea el Régimen MYPE Tributario del Impuesto a la Renta. Normas Legales - Perú. Lima, Perú: Normas Legales.

D.L.-№774. (8 de Diciembre de 2004). Ley de Impuesto a la Renta. Obtenido de https://www.perupetro.com.pe/wps/wcm/connect/corporativo/9d6e072c-6ae4-41ef-b4b4d38ae0a94965/3_Decreto_Legislativo_774.pdf?MOD=AJPERES

D.S.NN002-2017-PRODUCE. (2017 de Febrero de 01). Reglamento de Organización y Funciones del Ministerio de la Producción. Lima, Lima, Perú. Obtenido de https://www.produce.gob.pe/produce/descarga/dispositivos-legales/82661_1.pdf

D.S.-No122-94-EF. (19 de Setiembre de 1994). Reglamento de la Ley del Impuesto a la Renta. Obtenido de http://www.sunat.gob.pe/legislacion/renta/regla/

D.S.-No179-2004-EF. (8 de Diciembre de 2004). Téxto Único Ordenado de la Ley deo Impuesto a la Renta. Obtenido de http://www.sunat.gob.pe/legislacion/renta/ley/ 
Dirección Regional de Agricultura Tacna. (2017). Tacna Serie Histórica Producción Agrícola 2008 - 2017. Obtenido de

http://www.agritacna.gob.pe/gestores/estadistica/of_ol_estadidet_e/archivos/6506238174_13 81787781.pdf

Don, M. C., \& Milordo, J. M. (2010). Trabajo de investigación. Herramientas de gestión de la actividad vitícola. Facultad de Ciencias Económicas. Mendoza, Argentina. Obtenido de https://core.ac.uk/download/pdf/61887075.pdf.

Economía Simple. (2018). Obtenido de https://www.economiasimple.net/glosario/precio

Economía y Negocios. (2019). Pedro Cuenca: "Consumo de vino cre en el Perú". Obtenido de http://economiaynegocio.com/2019/03/13/consumo-de-vino-crece-en-el-peru/

El Peruano. Normas Legales. (15 de Junio de 2016). Normas Legales. Es la ley de Difusión del Vino y Pisco peruano. Obtenido de http://www.leyes.congreso.gob.pe/Documentos/Leyes/30460.pdf

Horngren, C. (2012). Contabilidad de costos Un enfoque gerencial. (P. Educación., Editor) Obtenido de https://profefily.com/wp-content/uploads/2017/12/Contabilidad-de-costos-Charles-T.Horngren.pdf

INDECOPI. (2011). Instituto Nacional de Defensa de la Competencia y de la Protección de la Propiedad Intelectual [INDECOPI] . Bebidas alcohólicas vitivinícolas. Vinos. Requisitos. Lima. Obtenido de https://kupdf.net/download/ntp-212-014-2011_596d686cdc0d60ca67a88e7b_pdf

Ley 30460 de promoción y difusión del vino y pisco peruano. (2016). Obtenido de Diario El Peruano: https://busquedas.elperuano.pe/normaslegales/ley-que-declara-de-interes-nacional-lapromocion-y-difusion-ley-n-30460-1392947-2

Martínez B., D. A., \& Benitez B., K. (2013). Trabajo de Grado, Diseño del sistema de costeo basado en actividades ( $a b c$ ) en la empresa vinos Don José fabricados por Onofre, ubicada en el municipio de Bolívar Valle del Cauca. Obtenido de Universidad del Valle. Facultad de Ciencias de la Administración. Programa Académico de Contaduría: http://bibliotecadigital.univalle.edu.co/bitstream/10893/10977/1/0497183\%20.pdf

Ministerio de Agricultura y Riego [MINAGRI]. (2016). Boletín Estadístico de Producción Agrícola, Pecuaria y Avícola - Diciembre 2016. Ministerio de Agicultura y Riego. Obtenido de http://siea.minagri.gob.pe/siea/?q=publicaciones/boletines-estadisticos-2016-2017

Ministerio de Agricultura y Riego. (2008). Informe de registro de productores de uva en las regiones de Ica, Arequipa, Tacna y Lima provincias. Obtenido de http://minagri.gob.pe/portal/download/pdf/herramientas/boletines/DocumentoFinalVid.pdf

Möller, G. (2011). Repositorio Documental Universidad de Valladolid. Obtenido de http://uvadoc.uva.es/handle/10324/849

Paz, M. (2016). Repositorio Universidad Cesar Vallejo. Obtenido de http://repositorio.ucv.edu.pe/bitstream/handle/UCV/368/paz_vm.pdf

Perucontable. (Agosto de 2017). Perucontable. Obtenido de Ventajas-y-Desventajas-del-Costeo-ABC: https://www.perucontable.com/contabilidad/ventajas-y-desventajas-del-costeo-abc/

PRODUCE. (2017). Dirección Regional de Producción Tacna. Padrón Manufacturero Industrial de Tacna. Tacna, Perú.

Región Tacna. (2017). Ocho empresas compitieron por presentar el mejor vino de Tacna. Obtenido de http://www.regiontacna.gob.pe/visor/noticia/NO00006700

Sánchez, B. (2013). Implicancias del método de costeo ABC. Quipukamayoc, 21(39), 65-73. Obtenido de http://revistasinvestigacion.unmsm.edu.pe/index.php/quipu/article/view/6273

Vaca López, A. (2012). Los sistemas de costeo: bases y metodologías. (C. U. Humboldt., Ed.) Obtenido de https://revistas.ugca.edu.co/index.php/contexto/article/view/34/54

Vinicultura. (Setiembre de 2014). Despalillado de la uva. Obtenido de http://www.vitivinicultura.net/despalillado-de-la-uva.html\#Que_es_el_despalillado_de_la_uva

Vino, C. d. (Enero de 2011). Recepción de la Vendimia. Obtenido de https://www.campusdelvino.com/blog/item/80-vendimia-uva-vino

Vitivinícola. (octubre de 2018). Obtenido de Filtracion en enologia para lograr vinos limpios: https://www.interempresas.net/Vitivinicola/Articulos/226836-Filtracion-en-enologia-paralograr-vinos-limpios.html 